\title{
Accelerating Rare Disease Drug Development: Lessons Learned from Muscular Dystrophy Patient Advocacy Groups
}

\author{
Raymond A. Huml, MS, DVM, RAC ${ }^{1}$ (1) - Jill Dawson, $\mathrm{PhD}^{2}$ - Michelle Bailey, $\mathrm{MD}^{1}$ • Nermina Nakas, MD, MPH ${ }^{1}$. \\ Jane Williams, MD, MPH ${ }^{1}$. Maryna Kolochavina, PharmD, PMP ${ }^{1}$. Jonathan R. Huml, BA, BSc ${ }^{3}$
}

Received: 1 July 2020 / Accepted: 9 September 2020 / Published online: 24 September 2020

(c) The Drug Information Association, Inc 2020

\begin{abstract}
With scientific and molecular advancements related to disease pathogenesis, advances in gene and stem cell therapies, and the promise of lucrative markets for biopharmaceutical companies, there has been a rapid expansion in the number of potential new muscular dystrophy (MD) treatments. The first champion for a newly diagnosed MD patient and their caregivers is typically an MD-specific patient advocacy group (PAG). Muscular dystrophy PAGs have been among the most active in the rare disease drug development space. Notable achievements in the last decade include promulgating the first U.S. clinical research guidance, setting up registries and natural history studies, and investing in companies—-some of which have brought potentially disease-modifying products to the market. This paper will discuss five key strategies that have been successfully employed by MD PAGs to advance treatments: (1) creating a national registry, (2) understanding the barriers to identifying patients with certain subtypes of muscular dystrophy to participate in clinical trials, (3) partnering with the biopharmaceutical industry, (4) collaborating with the regulators, and (5) incorporating market access and use insights early in clinical development. While clearly helpful within the MD community, these tactics could also be employed by PAGs representing other types of rare diseases.
\end{abstract}

Keywords Rare disease drug development $\cdot$ Muscular dystrophy $\cdot$ Patient advocacy

\section{Background: Muscular Dystrophies}

Rare diseases were once a neglected aspect of pharmaceutical research, relegated to non-profits and philanthropic projects. Today, with new blockbuster drugs being few and far between, and regulators incentivizing drug developers to pursue unmet needs, drug targets are increasingly being elucidated and companies are investing billions in rare disease research-with impressive results. Gene and stem cell therapies are the breakthrough treatments for a majority of rare diseases. Examples include a gene therapy treatment for spinal muscular atrophy (SMA), an ultra-rare neuromuscular disease, in children under two years of age (AveXis'

Raymond A. Huml

raymond.huml@ syneoshealth.com

Syneos Health ${ }^{\circledR}$, Morrisville, NC, USA

2 Danville, CA, USA

3 University of North Carolina at Chapel Hill, Chapel Hill, NC, USA
Zolgensma $\left.{ }^{\circledR}\right)$, and the first gene therapy for an inherited retinal disease (Spark Therapeutics' Luxturna ${ }^{\circledR}$ ).

Muscular dystrophy (MD) - a group of inherited, rare, degenerative muscle diseases that cause progressive muscle weakness due to defects in genes affecting the expression of muscle proteins- has a range of complex features that make treatments elusive. Like other rare diseases, the muscular dystrophies affect small and often widely scattered populations. For example, Duchenne Muscular Dystrophy (DMD) has an estimated incidence of 1 in 5000 live births [1]. This makes the recruitment of patients in rare disease trials challenging. These conditions typically lack natural history data, suitable non-clinical data, animal models, regulatory guidance, and clear primary and secondary endpoints and biomarkers for clinical trials, given the genotypic and phenotypic variability often seen within a single condition. The prognosis for the various MDs ranges from premature death in infancy, seen in congenital Myotonic Dystrophy type 1 (DM1), to onset in the third to sixth decades as in the case of mild Myotonic Dystrophy type 2 (DM2), which has a normal life expectancy [2]. New treatments, especially 
ones that are disease-modifying (slowing down or halting disease progression) or curative (restoring normal function) are desperately needed for these rare diseases.

With scientific and molecular advancements related to disease pathogenesis, advances in gene and stem cell therapies, and the promise of lucrative markets for biopharma companies, there has been a rapid expansion in the number of potential new MD treatments. See Table 1 below for a list of clinical trials posted on Clinicaltrials.gov by type of muscular dystrophy.

Myotonic Dystrophy (DM), with a prevalence of 0.5 to 18.1 per 100,000 , is the most common type of MD, followed by Facioscapulohumeral MD (FSHD) and Duchenne MD (DMD) [4, 5]. Of note, Limb-Girdle MD (LGMD) has an estimated minimum prevalence of 1 in 20,000 [6]. According to Orpha.net, [7] a portal for rare diseases and orphan drugs, FSHD prevalence is estimated at 4.5 cases $/ 100,000$ persons (using a European database), but can range from 1 to $9 / 100,000$ persons (globally). [8].

Although no therapies proven to stop or reverse the progression of MDs have been approved, several companies are pursing potential treatments for MD and have advanced to Phase II and III clinical trials. Gene therapies are under early clinical investigation, with a handful of Phase I trials. Only three products, golodirsen (Sarepta's Vyondys 53), eteplirsen (Sarepta's Exondys 51 ${ }^{\mathrm{TM}}$ ) and deflazacort (PTC Therapeutics' Emflaza®), have been approved for the treatment of DMD in the United States (U.S.), while deflazacort and ataluren (PTC Therapeutics' Translarna ${ }^{\mathrm{TM}}$ ) are approved to treat DMD in the European Economic Area.

\section{Introduction to the Patient Advocacy Groups}

The first champion for a newly diagnosed MD patient and their caregivers is typically an MD-specific patient advocacy group (PAG) [9]. In the U.S., the first point of call for information

Table 1 Muscular Dystrophy Types as Referenced on Clinicaltrials. gov [3].

\begin{tabular}{lc}
\hline Type of Muscular Dystrophy & $\begin{array}{c}\text { Number of } \\
\text { References }\end{array}$ \\
\hline Duchenne MD (DMD) & 272 \\
Becker MD (BMD) & 268 \\
Myotonic Dystrophy (DM) & 52 \\
Limb-Girdle MD (LGMD) & 34 \\
Facioscapulohumeral MD (FSHD) & 32 \\
Congenital MD (CMD) & 17 \\
Distal MD (DD) & 16 \\
Oculopharyngeal MD (OPMD) & 12 \\
Emery-Dreifuss MD (EDMD) & 1 \\
\hline
\end{tabular}

and support is typically the Muscular Dystrophy Association (MDA) or one of the disease-specific groups for several of the nine forms of MD shown in Table 2.

The MDA is the U.S.' largest non-profit supporter of research on over 40 different neuromuscular diseases, and has invested more than $\$ 1$ billion in funding since its inception. The organization supports families affected by MD through patient advocacy, fundraising, local engagement, and comprehensive health care services.

Some PAGs are large, well-known and well-funded. Others, such as those representing patients with extremely rare subtypes of MD, may have few members. Other subtypes may have no formal PAG. As in other rare diseases, some MD PAGs were started by parents of children diagnosed with MD.

This paper will discuss five key strategies that have been successfully employed by MD PAGs to advance treatments. While clearly helpful within the MD community, these tactics could also be employed by PAGs representing other types of rare diseases:

1. Create a national registry to identify all patients with a particular disease. Case study: MDA's NeuroMuscular ObserVational Research (MOVR) Data Hub

a. Understand the natural history of the disease (or subset of the disease), or how it progresses without intervention in order to determine if a proposed therapy is working/having an effect

2. Understand the barriers to identifying patients with certain subtypes of muscular dystrophy to participate in clinical trials

a. Learn from patients first-hand about their needs, in order to identify and prioritize disease-modifying treatment targets

3. Partner with the biopharmaceutical industry to ensure that patient needs are taken into account in clinical trials

4. Collaborate with regulators to develop disease-specific regulatory guidance, decreasing the risk associated with rare disease drug development

5. Incorporate market access and use insights early in clinical development, decreasing the risk of partial or conditional coverage after approval

Each of these strategies is discussed below.

\section{Create a National Registry}

Patient registries are defined by the Agency for Healthcare Research and Quality (AHRQ) as organized systems that use observational research methods to collect data for the 
Table 2 Select Patient Advocacy Groups for MD.

\begin{tabular}{|c|c|}
\hline Type of MD & Sources of Information \& Support \\
\hline Duchenne MD (DMD) & $\begin{array}{l}\text { Muscular Dystrophy Association (MDA, mda.org) } \\
\text { Parent Project Muscular Dystrophy (PPMD, https://www.parentprojectmd.org) } \\
\text { The Foundation to Eradicate Duchenne (https://duchennemd.org) } \\
\text { Duchenne Alliance (https://www.duchennealliance.org) } \\
\text { Other organizations around the world are listed at https://www.treat-nmd.eu/dmd/patient-organizations/, } \\
\text { https://www.duchenneconnect.org, \& https://www.cureduchenne.org }\end{array}$ \\
\hline Becker MD (BMD) & MDA (https://mda.org/disease/becker-muscular-dystrophy) \\
\hline Congenital MD (CMD) & $\begin{array}{l}\text { MDA (mda.org) } \\
\text { Cure CMD (https://curecmd.org) }\end{array}$ \\
\hline Distal MD (DD) & MDA (https://mda.org/disease/distal-muscular-dystrophy/types) \\
\hline Emery-Dreifuss MD (EDMD) & $\begin{array}{l}\text { MDA (https://www.mda.org/disease/emery-dreifuss-muscular-dystrophy) } \\
\text { National Organization for Rare Disorders (NORD, https://www.rarediseases.org; https://www.rarediseas } \\
\text { es.org/rare-disease-information/rare-diseases/byID/590/viewAbstract) }\end{array}$ \\
\hline Facioscapulohumeral MD (FSHD) & $\begin{array}{l}\text { FSHD Society (https://www.fshdsociety.org) } \\
\text { MDA (mda.org) } \\
\text { Friends of FSH Research (https://www.fshfriends.org) }\end{array}$ \\
\hline Limb-Girdle MD (LGMD) & $\begin{array}{l}\text { MDA (mda.org) } \\
\text { Jain Foundation (https://www.jain-foundation.org) } \\
\text { Coalition to Cure Calpain } 3 \text { (https://www.curecalpain3.org/) } \\
\text { The Speak Foundation (https://thespeakfoundation.com/) }\end{array}$ \\
\hline Myotonic Dystrophy (DM) & $\begin{array}{l}\text { MDA (mda.org) } \\
\text { Myotonic Dystrophy Foundation (https://www.myotonic.org) }\end{array}$ \\
\hline Oculopharyngeal MD (OPMD) & $\begin{array}{l}\text { MDA (https://mda.org/disease/oculopharyngeal-muscular-dystrophy) } \\
\text { NORD (https://www.rarediseases.org/rare-disease-information/rare-diseases/byID/1182/viewAbstract) }\end{array}$ \\
\hline
\end{tabular}

scientific assessment of patient outcomes [10]. Registries can vary in sophistication and scope, from a simple MS Excel spreadsheet compiled by a single physician to complex international databases that can be accessed online across multiple institutions. Registries are simpler to set up than an electronic medical record, which keeps track of all the patients a doctor follows. A registry only keeps track of a small subpopulation of patients with a specific condition.

Registries are needed to help pharmaceutical and biotechnology companies identify patients for regional and global trials, expedite drug development, protect patients' rights, [11] and help meet patient expectations. Examples of patient expectations could include: being notified of new clinical trials for potential enrollment, protection of data privacy (e.g., privacy rules pertaining to the U.S. Health Insurance Portability and Accountability Act of 1996), and being included in the clinical trial design process by recommending suitable Patient-Reported Outcomes (PROs).

Rapid identification of MD patients is vital for information sharing, advocacy, and to increase awareness of opportunities to advance scientific knowledge about these conditions. Once therapies are approved, registries also have a role in post-marketing surveillance [11].

In recognition of the difficulties of identifying trial participants in rare diseases and the negative impact of this on research progress, the MDA is working with other patient advocacy groups and MDA Care Centers to provide a world map of people with myopathies. In 2018, the MDA announced that it would revamp its U.S. Neuromuscular Disease Registry to create a NeuroMuscular ObserVational Research (MOVR) data hub [12]. The MDA will use the MOVR data hub, among other tools, to study the natural history of muscular dystrophy and related muscle diseases, collect information on practice patterns, inform care guidelines and improve quality of care for patients.

According to the FDA, [13] "The natural history of a disease is traditionally defined as the course a disease takes in the absence of intervention in individuals with the disease, from the disease's onset until either the disease's resolution or the individual's death. A natural history study is a preplanned observational study intended to track the course of the disease. Its purpose is to identify demographic, genetic, environmental, and other variables (e.g., treatment modalities, concomitant medications) that correlate with the disease's development and outcomes. Natural history studies are likely to include patients receiving the current standard of care and/or emergent care, which may alter some manifestations of the disease."

This type of study can provide rare disease treatment sponsors with an understanding of how the target disease progresses and an objective way to determine whether a proposed therapy is working. Natural history studies are essential to identify the correct patient populations, identify and develop outcomes assessments for inclusion into research and 
development protocols, and also to identify and develop biomarkers to be used for early knowledge generation and early confirmation of value proposition [13]. Natural history studies can potentially serve as a comparator arm in single-arm clinical trials, taking the place of a placebo arm.

All drug development programs benefit from a firm scientific foundation, including an understanding of the natural history of the disease. The natural history of rare diseases is often poorly understood, and the need for prospectively designed, protocol-driven natural history studies initiated in the earliest drug development planning stages cannot be overemphasized.

Although the FDA does not require natural history studies, the agency recommends that industry evaluates the depth and quality of existing natural history knowledge at an early stage, to determine whether this is sufficient to inform drug development programs. A natural history study initiated early may run in parallel with the initial stages of drug development—including preclinical development—and may allow updating of drug development strategies as new learning emerges. Including patients and families early on in the protocol development process can help to inform the study design.

Much of the data on natural history of rare diseases is collected by PAGs. For example, in December 2016, CureDuchenne Ventures-the investment arm of the DMD PAG, CureDuchenne-invested in Therapeutic Research in Neuromuscular Disorders Solutions (TRiNDS), a contract research organization (CRO) born from the Cooperative International Neuromuscular Research Group (CINRG), an academic sponsor. According to the TRiNDS Website, "CINRG has established the largest and longest Duchenne natural history study to date." $[14,15]$.

In a second example, patients with LGMD (with over 35 different subtypes identified to date) were invited at the First National Limb Girdle MD Conference in Chicago in 2019 to enroll in the "Defining Clinical Endpoints in Limb Girdle Muscular Dystrophy" or GRASP study. This aims to better understand the natural history of LGMD as well as define the best endpoints to study in clinical trials [16]. Another example could include use of a natural history study to record a baseline period prior to the set-up of a gene therapy dose escalation (first-in-human) clinical study. [17].

Implementing a patient-centric approach to therapeutic development for rare diseases is proving helpful in ensuring maximum patient benefits [18].

\section{Understand the Barriers to Identifying Patients}

There are multiple barriers to identifying patients with rare diseases. First, the cost to obtain a diagnosis may be prohibitive. For example, in the U.S., until just recently, it would cost over $\$ 2000$ for some patients with FSHD to obtain a diagnosis. Secondly, due to U.S. insurance discrimination practices, MD patients with mild disease (e.g., Becker's or FSHD patients) may not wish to be diagnosed. Other barriers include:

- MD patients are not all treated at specialized centers

- "Big data" is of value for more prevalent diseases, but for rare diseases with small patient populations, there may not be sufficient information to benefit from artificial intelligence (AI)

- Issues regarding testing and diagnosis may prevent identification.

In the U.S., most patients with MD are diagnosed by a neurologist or pediatric neurologist, and seen by a wide variety of healthcare specialists who provide multidisciplinary comprehensive care at MDA Care Centers. General Practitioners (GPs) and/or pediatricians may treat patients with milder forms of MD.

After the MDA Care Centers, the specific MD PAGs may be the next best source of registries and data collected on patients. For example, Parent Project Muscular Dystrophy (PPMD) has a 10-year DMD registry which contains over 5000 patients diagnosed with DMD, [19] and the FSHD Society, with support from the MDA, has the FSHD Clinical Trials Research Network (CTRN), a consortium of academic research centers [20].

Because there are so few patients available for natural history studies and clinical trial participation, all sources of data should be explored when trying to identify patients with rare diseases or subsets of rare diseases.

"Big data" can be of value for improving our understanding of rare disease patient populations. This is especially true if a "digital footprint" - the trail of medical records (which may include electronic health record data, laboratory data, imaging data, physician-entry [notes] data, etc.) that accompanies each de-identified patient in a healthcare database - can be ascertained. Inconsistencies in sampling quality may limit the utility of data at present, however.

The use of digital technologies, AI applications and big data in rare disease populations has helped drive a focus on potential ethical and legal aspects of data governance [21]. While the FDA has issued guidance documents [22, 23] related to Clinical Decision Support software, AI and machine learning, there has been very little guidance on risk monitoring related to AI use in the design of clinical trials, particularly where there is a high unmet need for product innovation. It is important to ensure the proper informed consent (including assent for vulnerable pediatric populations) and responsible use of AI technology, so that any potential harms are made clear to all involved. 
In some cases, ICD-10 codes are unavailable or ambiguous (for example, multiple rare diseases may be classified by one code, Duchenne and Becker's MD are classified together, and there is no unique ICD-10 code for LGMD) or there may be no prescription therapies for a particular rare disease. In these cases, Electronic Health medical Record (EHR), medical claims and prescription data may be less useful [24]. Nonetheless, the knowledge of PAGs, key opinion leaders (KOLs) at academic centers, and registry databases may still be useful adjunct sources of data to identify patients with rare diseases.

There is also a risk that rare disease data may be biased due to self-selection. MD PAGs have highlighted that some patients with milder forms of MD (e.g., Becker's MD, late onset FSHD, etc.) may wish not to be diagnosed due to discriminatory insurance practices in the U.S., or may be dissuaded from obtaining a molecular diagnosis due to its high cost or lack of insurance coverage.

In addition, sample collection for MD diagnosis may be onerous or painful, such as venipuncture (for blood samples) and/or muscle biopsies. The authors have seen a trend toward ultrasound-guided needle biopsies (which require less muscle tissue and heal quicker), whereas in the past, punch muscular biopsies were utilized (which were less specific, removed more tissue, were often more painful, and sometimes required stitches). The advances in diagnostic technologies and availability of less invasive approaches has in some cases shortened the odyssey for rare disease diagnosis, particularly in the pediatric population.

\section{Learn from Patients First-Hand}

Sponsors of new treatments for patients with MD may decide to pursue a treatment that does not directly address the perceived mechanism of action or allow for a chance for a cure. For example, rather than "fix" a dysregulated protein, such a dystrophin or sarcoglycan, a sponsor may wish to target control of a symptom that has been defined as clinically significant by patients with MD.

Absent a cure, sponsors of rare disease treatments need to determine the potential balance between treatment benefits and risks that may be acceptable to patients and caregivers. For example, what is the ranking importance of decreasing fatigue, being able to breathe better, having an increased range of motion, or having an improved mood or decreased anxiety?

In the U.S., this type of patient/caregiver feedback can be obtained via a Patient-Focused Drug Development (PFDD) meeting with the FDA. The FDA encourages involvement of patients, their caregivers, and advocates in the rare disease drug development process. PFDD integrates patient input into medical product development and decision making, starting in the translational phase and continuing all the way through post-marketing. According to the FDA website, patient-focused drug development "is a systematic approach to help ensure that patients' experiences, perspectives, needs, and priorities are captured and meaningfully incorporated into drug development and evaluation. As experts in what it is like to live with their condition, patients are uniquely positioned to inform the understanding of the therapeutic context for drug development and evaluation" [25].

Patient-reported outcomes (PROs) are being developed based on patient preferences and input. During the review of an investigational drug, patients and caregivers can provide important information about their experiences, perspectives, needs, and priorities related to potential endpoints and meaningful outcomes. For industry, this input is also being sought through public websites such as The Duchenne Xchange (see https://www.duchennexchange.org/). Sponsored by both PAGs and industry, this solicits feedback from both patients and caregivers on topics such as clinical trial protocols to identify barriers to recruitment prior to implementation of a clinical trial.

Other MD PAGs have private Facebook chat rooms and other ways to solicit input from patients (e.g., FSHD Society).

\section{Partner with the Biopharmaceutical Industry}

PAGs are the trusted partners with patients and their caregivers. As such, they have established reputations in the U.S. as a protector of patients and have an important role in disseminating data and new ways of thinking at conferences, via presentations, abstracts, and posters (including virtual approaches during the COVID-19 pandemic). They can help sponsors identify patients and develop suitable endpoints for clinical trials, in light of evolving regulatory guidance, or where such guidance does not exist. All MD PAGs could benefit from collaboration and partnership with industry by sharing capital, insights, expertise and, in some cases, drug development risk.

Case examples of such mutually beneficial relationships that help drive rare disease drug development include:

- The MDA announced that it would help expand the Facioscapulohumeral Muscular Dystrophy Clinical Research Network investing in seven medical centers that specialize in FSHD research and clinical care. MDA's clinical research grant was designed to be provided over three years to develop and maintain a core FSHD Clinical Trial Research Network (CTRN). The goal of the research network is to spur advances in FSHD and expedite the development of new therapies [26]. 
- PPMD announced a partnership with Sarepta to launch the Duchenne Outcomes Research Interchange. The interchange will combine data from the Duchenne Registry with clinician-reported, post-marketing surveillance data provided by industry partners with approved therapies. The Duchenne Outcomes Research Interchange is designed to provide critical information to clinicians, sponsors, payers, regulators, and the patients/families who participate in the Duchenne Registry [27].

- Fulcrum announced that it had raised $\$ 80$ million in a Series B round led by Foresite Capital and other institutions. Fulcrum will use the proceeds to advance its lead program in FSHD into clinical testing, and to progress its pipeline of therapeutics for rare, genetically-based neuromuscular, central nervous system and hematologic disorders. The company has initiated clinical trial readiness studies in FSHD in partnership with the FSHD Clinical Trial Research Network in order to standardize a set of tools and measurements for Fulcrum's future clinical drug trials [28].

- North Carolina-based Bamboo Therapeutics received $\$ 49.5$ million in what appeared to be a Series A equityfinancing round. Bamboo was founded based on the work of the first researcher to use the adeno-associated virus $(\mathrm{AAV})$ as a gene therapy vector, re-engineering the virus to "target delivery to certain tissues, de-target other tissues, and improve its safety," according to the company's website. Bamboo announced that CureDuchenne Ventures LLC had invested an undisclosed amount of money in the company. The company's gene therapy had shown some positive effects in animal models with DMD [29]. After this announcement, Bamboo Therapeutics was acquired by Pfizer [30].

\section{Collaborate with Regulators to Develop Disease-Specific Regulatory Guidance}

Sponsors of rare disease therapies-in common with other sponsors-benefit from regulatory guidance that is diseasespecific, and therefore minimizes regulatory risk. For rare diseases that lack specific regulatory guidance, PAGs should consider developing their own proposed guidance to share with regulators. In one success story, in June 2014, PPMD and more than 80 representatives of the DMD community broke new ground when they submitted the first-ever patient advocacy-initiated draft guidance to the FDA. The submission, made at the invitation of the FDA, was titled "Guidance for Industry: Duchenne Muscular Dystrophy, Developing Drugs for Treatment over the Spectrum of Disease." The draft guidance sought to address unmet clinical needs of individuals with DMD and to accelerate development and review of safe and effective therapies for this illness [31].
Short of developing regulatory guidance, FDA's PFDD option allows PAGs to summarize the patient perspective and educate the FDA [32].

\section{Working with the Regulators to Determine the Best Clinical Trial Endpoints}

Because the PAGs are often the best source of clinical information about the patients they represent, it is important to include their perspectives when designing clinical trials. PAGs frequently work with regulators, industry, and entities such as the Critical Path Institute (CPI), to identify the best primary and secondary endpoints for clinical trials. Once identified and tested, these endpoints must be validated by regulators for use in trials.

The CPI is a non-profit organization that specializes in leading public-private partnerships-called consortia-to develop drug development tools, and works toward qualification/endorsement of such tools with U.S. and EU regulatory agencies (e.g., FDA and EMA). Each consortium is supported by multiple industry stakeholders, which may include biopharmaceutical companies and clinical research organizations (CROs), and is advised by an FDA liaison to ensure that products of the consortia are suitable for qualification.

As an example, the CPI set up a Duchenne Regulatory Science Consortium to develop tools to accelerate therapy development for DMD [33]. Three key deliverables for this initiative include:

1. A Clinical Data Interchange Standards Consortium (CDISC) standard for DMD-which defines the regulatory acceptable format, structure and terminology used in databases from clinical studies

2. An integrated database collating natural history of disease data

3. A clinical trial simulation tool developed based on mathematical models of disease progression.

Further details related to DMD drug development are also provided by academia, working with the biopharma industry and PAGs.

\section{Incorporating Commercial Insights Early into a MD Research and Development Program}

Obtaining FDA approval or EU market authorization does not guarantee that payers will reimburse the product. The authors have seen examples where drugs approved for MD treatment were given conditional coverage or were restricted 
to a certain small group of patients based on age or level of function [34].

Growing concerns regarding the gap between the demand for health services and technologies and the available resources have driven introduction of systems to assess robust information around clinical benefit, value for money and potential adoption/diffusion and affordability of therapies coming to market.

There is disagreement between regulators and payers in terms of comparators, endpoints, and what the overall efficacy and safety package should look like [35]. Payers and Health Technology Assessment (HTA) boards recognize the value of integrated evidence. However, real-world evidence (RWE) used to inform pharmacy and therapeutic (P\&T) decisions varies from organization to organization and is limited. According to the 2017 European Working Group for Value Assessment and Funding Processes in Rare Diseases (ORPH-VAL), [36] "Many factors affect Orphan Medicinal Product (OMP) uptake, but one of the most important is the difficulty of making pricing and reimbursement $(\mathrm{P} \& \mathrm{R})$ decisions in rare diseases. Until now, there has been little consensus on the most appropriate assessment criteria, perspective or appraisal process." In the authors' experience, U.S. payers integrate natural history studies and registries to inform safety monitoring, utilization management and cost analysis, [37] and will consider RWE in label extensions and post-marketing surveillance. Reimbursement assessment processes consist of compiling, analyzing, assessing, and appraising the evidence available to show whether the health and economic benefits of a product compared to the standard of care- or current method of treatment-are sufficient to justify the price, above and beyond the requirements of regulatory authorities. This is especially important if there is evidence of cost savings (e.g., reduced hospitalizations and treatments, Global Health Economics \& Outcomes Research (GHEOR) and resource utilization) available or net improvement in outcomes meaningful to patients and treating physicians. A lack of understanding and implementation of these considerations early in clinical development can lead to a situation where sponsors of MD treatments struggle to gain reimbursement.

\section{Conclusion}

Muscular dystrophy PAGs have been among the most active and influential in the rare disease drug development space. Notable U.S. achievements in the last decade include promulgating the first FDA clinical research guidance, setting up registries and natural history studies, and investing in companies-some of which have brought potentially diseasemodifying products to the market.
As discussed in this paper, five key strategies to accelerate rare disease drug development have successfully been used in the MD drug development ecosystem. Based on successful use by MD-related PAGs to advance therapies for patients, these tactics could also be applied by other rare disease PAGs to achieve the same goals.

While some MD PAGS, such as MDA, receive '\$100 m in funding on a yearly basis, [38] enabling them to support larger projects such as the national registry, smaller groups such as FSHD Society, who only receive $>\$ 10$ m each year in funding [39], can provide equally important insights into trial design and patient needs. The MD PAGs have shown that it takes a collective to enable us all to better understand the disease and the research directions needed to advance optimal treatment options for patients.

\section{Acknowledgement}

The authors would like to thank Meredith L. Huml, Communications Assistant from Disability Rights of North Carolina, for her patient advocacy contributions and editorial insights.

\section{Author Contributions}

All authors had substantial contributions to the conception or design of the work, the acquisition, analysis, or interpretation of data for the work. All authors helped draft, revise the manuscript critically for important intellectual content, and provided approval for the final version. All authors agreed to be accountable for all aspects of the work in ensuring that questions related to the accuracy or integrity of any part of the work are appropriately investigated and resolved.

\section{Funding}

No author has any financial holdings or considerations to disclose.

\section{Compliance with Ethical Standards}

\section{Conflict of interest}

The authors have nothing to disclose.

\section{References}

1. Mendell JR, Shilling C, et al. Evidence-based path to newborn screening for Duchenne muscular dystrophy. Ann Neurol. 2012;71(3):304-13.

2. Turner C, Hilton-Jones D. Myotonic dystrophy: diagnosis, management and new therapies. Curr Opin Neurol. 2014;7(5):599606. https://doi.org/10.1097/WCO.0000000000000128.

3. Clinicaltrials.gov. US National Library of Medicine, National Institutes of Health at https://clinicaltrials.gov/ct2/home. Accessed 23 Mar 2020

4. Theadom A, Rodrigues M, Roxburgh R, et al. Prevalence of muscular dystrophies: a systematic literature review. Neuroepidemiology. 2014;43(3-4):259-68. https://doi.org/10.1159/000369343. 
5. Mercuri E, Bönnemann CG, Muntoni F. Muscular dystrophies. Lancet. 2019;394(10213):2025-38. https://doi.org/10.1016/S0140 -6736(19)32910-1.

6. Wicklund MP, Kissel JT. The limb-girdle muscular dystrophies. Neurol Clin. 2014;32:729.

7. Prevalence of rare diseases: bibliographic data, www.orpha.net, May 2014 Number 1, Orphanet report series. https://www.orpha .net/orphacom/cahiers/docs/GB/Prevalence_of_rare_diseases_by_ alphabetical_list.pdf. Accessed 30 Mar 2020.

8. Palmer E. Global Duchenne muscular dystrophy treatment market to see spectacular growth by 2019, says GlobalData, 14 Apr 2015 at https://www.fiercepharma.com/pharma/global-duchennemuscular-dystrophy-treatment-market-to-see-spectacular-growt h-by-2019-says. Accessed 30 Mar 2020.

9. Huml RA. Muscular Dystrophy: A Concise Guide. Berlin: Springer; 2016.

10. Gliklich RE, Levy D, Karl J, Leavy MB, Taylora T, Campion DM. Registry of patient registries (RoPR): project overview. Agency for Healthcare and Research Quality. May 2012.

11. Gliklick RE, Dreyer NA, Leavy MB, editors. Registries for evaluating patient outcomes: a user's guide (internet). 3rd Edition, Rockville, MD, Principles of Registry Ethics, Data Ownership, and Privacy, 2014 April 7.

12. Intersection between big data and therapeutic pipeline in neuromuscular disease will be life changing for 250,000 patients and caregivers, MDA website, 30 Oct 2018 at https://www.mda. org/press-releases/intersection-between-big-data-and-therapeuti c-pipeline-neuromuscular-disease-will-be. Accessed 30 Mar 2020.

13. Rare diseases: natural history studies for drug development, draft guidance for industry, 1 Mar 2019 at https://www.fda.gov/media /122425/download. Accessed 15 Jun 2020.

14. TRiNDS website at https://trinds.com/. Accessed 24 Mar 2020.

15. CureDuchenne ventures invests in TRiNDS to provide clinical trial solutions for neuromuscular disorders, 19 Dec 2016 at https ://www.cureduchenne.org/press-release/cureduchenne-venturesinvests-trinds-provide-clinical-trial-solutions-neuromuscular-disor ders/. Accessed 27 Mar 2020.

16. Defining clinical endpoints in Limb Girdle Muscular Dystrophy (LGMD) (GRASP); https://clinicaltrials.gov/ct2/show/NCT03 981289. Accessed 23 Mar 2020.

17. Natural History of Duchenne Muscular Dystrophy, ClinicalTrials. gov Identifier: NCT03882827, last updated posted 7 Jan 2020 at https://clinicaltrials.gov/ct2/show/NCT03882827. Accessed 15 Jun 2020.

18. Theme 4: when therapies meet the needs; 10th European Conference on rare disease \& orphan products (ECRD) 2020; 14-15 May 2020.

19. Parent project muscular dystrophy publishes 10 year registry report, 17 Jan 2019 at https://www.parentprojectmd.org/paren t-project-muscular-dystrophy-publishes-10-year-registry-repor t/. Accessed 24 Mar 2020.

20. Facioscapulohumeral muscular dystrophy clinical trial research network at https://www.kumc.edu/fshd.html. Accessed 24 Mar 2020.

21. Theme 6: the digital health revolution; 10th European Conference on Rare Disease \& Orphan Products (ECRD) 2020; 14-15 May 2020.

22. Clinical decision support software draft guidance for industry and food and drug administration staff, https://www.fda.gov/regulatory -information/search-fda-guidance-documents/clinical-decisionsupport-software.

23. Artificial intelligence and machine learning in software as a medical device, https://www.fda.gov/medical-devices/software-medic al-device-samd/artificial-intelligence-and-machine-learning-softw are-medical-device. Accessed on 12 Jun 2020.
24. Huml RA, Dawson J, Lipworth K, Rojas L, Warren J, Manaktala C, Huml JR. Use of big data to aid patient recruitment for clinical trials involving biosimilars and rare diseases, DIA's J Therapeut Innov Regulat Sci . Published online 11 Dec 2019.

25. FDA website: CDER patient-focused drug development, $21 \mathrm{Apr}$ 2020 at https://www.fda.gov/drugs/development-approval-proce ss-drugs/cder-patient-focused-drug-development. Accessed 17 Jun 2020.

26. MDA funds expansion of facioscapulohumeral muscular dystrophy clinical research network, 20 Jun 2018 at https://www.mda. org/press-releases/mda-funds-expansion-facioscapulohumeralmuscular-dystrophy-clinical-research-network. Accessed 27 Mar 2020.

27. PPMD \& Sarepta Partner to Launch Duchenne outcomes research interchange, 7 Sept 2018 at https://www.parentprojectmd.org/ ppmd-sarepta-partner-to-launch-duchenne-outcomes-researchinterchange/. Accessed 27 Mar 2020.

28. Fulcrum therapeutics raises $\$ 80$ million series B round for facioscapulohumeral muscular dystrophy (FSHD), 5 Sept 2018 at https://www.equities.com/news/fulcrum-therapeutics-raises-80million-series-b-round-for-facioscapulohumeral-muscular-dystr ophy-fshd. Accessed 27 Mar 2020.

29. Gene Therapy Company bamboo therapeutics raises $\$ 49.5$ million in apparent series a financing round, 15 Feb 2016 at https:// www.biospace.com/article/gene-therapy-company-bamboo-thera peutics-raises-49-5-million-in-apparent-series-a-financing-round -/. Accessed 27 Mar 2020.

30. Pfizer Press release: Pfizer aims to become industry leader in gene therapy with acquisition of Bamboo Therapeutics, Inc., 1 Aug 2016 at https://www.pfizer.com/news/press-release/press-relea se-detail/pfizer_aims_to_become_industry_leader_in_gene_thera py_with_aquisition_of_bamboo_therapeutics_inc. Accessed 17 Jun 2020.

31. Huml RA. Filling a regulatory void: patient advocates submit guidance for Duchenne muscular dystrophy, RAPS Regulatory Focus, 5pp; electronically posted to the RAPS Website on August 7, 2014.

32. CDER Patient-Focused Drug Development, FDA Website at https ://www.fda.gov/drugs/development-approval-process-drugs/cderpatient-focused-drug-development. Accessed 24 Mar 2020.

33. Larkindale J, Conrado DJ, Berg AK, et al. Development of clinical trial simulation tool for Duchenne Muscular Dystrophy through the Duchenne Registry Science Consortium (D-RSC). Critical Path Institute, poster presentation, 2019, Tucson, AZ.

34. Schaeffer S, Wrong again: why Sarepta's $\$ 300 \mathrm{k}$ price for DMD drug invalidates reasons for accelerated approval, Biocentury, September 16, 2016 at https://www.biocentury.com/article/27910 1/why-sarepta-s-300k-price-for-dmd-drug-invalidates-reasons-foraccelerated-approval. Accessed June 15, 2020.

35. Tafuri G, Pagnini M, Moseley J, et al. How aligned are the perspectives of EU regulators and the HTA bodies? A comparative analysis of regulatory HTA parallel scientific advice. Br J Clin Pharmacol. 2016;82(4):965-73.

36. Annemans L, Ayme S, Le Cam Y, et al. Recommendations for the European Working Group for Value Assessment and Funding Processes in Rare Diseases (ORPH-VAL). Orphanet J Rare Dis. 2017;12:50

37. Malone DC, Brown M, Hurwitz JT, et al. Real-world evidence: useful in the real world of US payer decision making? How? When? And what studies? Value Health. 2018;21(3):326-33.

38. MDA Website: Financial Information and Annual Reports at https ://www.mda.org/about-mda/financials. Accessed 24 Jun 2020.

39. FSHD Society Website: Financial Information and Donor Reports at https://www.fshdsociety.org/about-us/financials/. Accessed 24 Jun 2020. 